\title{
Problemas de segundo orden: hacia una actualización semántica de la crítica inmanente
}

\author{
ERIKa Martínez \\ Pontificia Universidad Católica del Perú
}

Resumen: El presente artículo tiene como finalidad entrecruzar la propuesta de Rahel Jaeggi de una crítica inmanente de las formas de vida con la teoría inferencialista de Robert Brandom. El proceso de racionalización de los recursos culturales y conceptuales de una comunidad de hablantes que desarrolla Brandom resultaría útil para comprender las formas de vida como instancias de resolución de problemas de segundo orden. Para ello, se explicará, en primer lugar, la noción de formas de vida como instancias de solución de problemas de segundo orden de Jaeggi -la que será complementada con la noción de "libertad expresiva" de Brandom. En segundo lugar, se desarrollará el inferencialismo de Robert Brandom y se buscará articular los problemas de segundo orden con la explicitación de las relaciones inferenciales de conceptos en un espacio de razones. Finalmente, se presentará la apertura del espacio de razones como el lugar donde se encuentra el germen de la crítica que podría dar pie a una transformación social.

Palabras clave: teoría crítica, crítica inmanente, inferencialismo, Brandom, Jaeggi.

Abstract: The purpose of this article is to crisscross Rahel Jaeggi's proposal of an immanent critique of forms of life with Robert Brandom's inferentialist theory. The process of rationalization of a community of speakers' cultural and conceptual resources developed by Brandom is useful to understand forms of life as solving instances of second-order problems. To this end, I will explain Jaeggi's notion of forms of life as solving instances of second-order problems, which I will complement with Brandom's "expressive freedom". Secondly, I will develop his inferentialist approach, which I will articulate with second-order problems in relation to the explanation of the inferential relations of concepts within a space of reasons. Finally, I will develop the argument in 
which the opening of a space of reasons may be understood as the place where one can find the germ of the critique that could lead to social transformation.

Keywords: critical theory, immanent critique, inferentialism, Brandom, Jaeggi.

\section{§ 1. Introducción}

El presente trabajo tiene como finalidad analizar y discutir la propuesta de Rahel Jaeggi sobre la realización de una crítica inmanente de las formas de vida en relación con la teoría inferencialista brandomiana. Las formas de vida son entendidas por Jaeggi como prácticas sociales formadas culturalmente, las que constituyen instancias de resolución de problemas de segundo orden. En relación con este último punto, resulta útil recurrir a Brandom, quien desarrolla una teoría del proceso de racionalización de los recursos culturales y conceptuales de una comunidad de hablantes, el cual es necesario para resolver los problemas de segundo orden.

Para Brandom, en torno a los enunciados existe una serie articulada de creencias y razones. En ese sentido, las conexiones inferenciales que hacen que derivemos de ciertos conceptos determinadas atribuciones o características tienen que ver con una lógica material, es decir, implican consecuencias prácticas. De esta manera, al explicitar el proceso del establecimiento de una relación entre los conceptos que una sociedad usa y las creencias que asume en función de estos, se presentaría el germen de una crítica que posibilitaría la transformación de la sociedad.

Según Jaeggi, la crítica es la búsqueda de un criterio mediante el cual uno puede reconocer si ciertas dinámicas se han convertido en procesos de aprendizaje. Estos consistirían en la superación dialéctica de contradicciones en los contenidos conceptuales, pues estos problemas tienen su origen en soluciones anteriores y las nuevas soluciones comprenderán estas últimas.

La argumentación se dividirá del siguiente modo. En primer lugar, se explicará la noción de formas de vida como instancias de solución de problemas de segundo orden de Rahel Jaeggi -la que será complementada con la noción de “libertad expresiva” de Robert Brandom. En segundo lugar, se desarrollará el 
inferencialismo de Robert Brandom, y se buscará articular los problemas de segundo orden tematizados por Jaeggi con la explicitación de las relaciones inferenciales de conceptos en un espacio de razones. Finalmente, se presentará la apertura del espacio de razones como el lugar donde se encuentra el germen de la crítica que podría dar pie a una transformación social.

\section{§ 2. Las formas de vida como objeto de la crítica inmanente}

En el artículo "Towards an Immanent Critique of Forms of Life” (2015), Rahel Jaeggi critica al liberalismo político por haberse presentado como "éticamente abstinente" frente a las formas de vida y haberlas relegado así al ámbito de lo privado, como si se tratara de meras preferencias o cuestiones referentes a la identidad que no deberían ser problematizadas; en contraste con la esfera pública, donde se establecen los temas de deliberación colectiva. Por ejemplo, en el liberalismo rawlsiano, la tematización de las formas de vida ha sido insuficiente para considerar a estas como problemáticas: se ha buscado hallar una forma armónica de coexistencia entre las diferentes ideas acerca del sentido de la vida. Sin embargo, una crítica de las formas de vida se vuelve necesaria en contextos contemporáneos marcados por el conflicto y la transformación social, en donde principios éticos que no habían sido cuestionados antes lo son ahora por nuevas tecnologías, o cuando determinadas prácticas sociales se vuelven problemáticas (Jaeggi 2015, 13).

La teoría crítica de la sociedad, dirá Jaeggi, ha estado comprometida desde sus inicios con esta empresa, sin caer en la neutralidad ética del liberalismo ni en la propuesta de una teoría perfeccionista acerca de la vida buena. Según ella, dicha teoría ha desarrollado una crítica de las formas de vida atenta a las condiciones necesarias para el logro de la autodeterminación individual y colectiva. Para Axel Honneth, el trasfondo común de todos los representantes de la teoría crítica ha sido, pues, la noción de que “(...) un proceso histórico de formación [racional] ha sido distorsionado por la situación social de una manera que solo puede corregirse en la práctica" $(2009,29)$, bajo la presuposición normativa de una "(...) constitución de las condiciones sociales que estaría intacta si garantizara a todos los miembros la oportunidad de lograr la autorrealización" $(2009,30)$. Es así que la teoría crítica se ha encontrado relacionada con un entendimiento de la crítica inmanente de las formas de vida 
basada en su relación con la comprensión de problemas y crisis -podríamos referirnos a las distorsiones del proceso de formación racional o "patologías sociales" de las que habla Honneth-que parten de reflexiones socio-ontológicas sobre la misma estructura de las formas de vida, es decir, sobre las prácticas y actitudes constitutivas de las mismas o su contenido intrínseco. Estas son susceptibles a la crítica debido a que se presupone la existencia de una racionalidad específica propia de cada forma de vida; así pues, uno puede discutir acerca de ellas con razones. Este punto será desarrollado en la tercera parte de este artículo.

Las formas de vida, según Jaeggi, se distinguen de las meras preferencias, en el sentido en que estas últimas no propician opiniones que posibiliten discusiones'. De esta manera, ella define las formas de vida como un "conjunto inerte de prácticas" $\mathrm{o}$, en otras palabras, órdenes de coexistencia humana culturalmente conformadas, que implican tanto "un entramado de prácticas y orientaciones" como la materialidad de sus expresiones (manifestaciones institucionales, sistemas jurídicos, etc.) (Jaeggi 20I5, 16).

Las prácticas sociales son, pues, “(...) secuencias de acciones singulares que pueden ser más o menos comprehensivas y complejas y que tienen un carácter más o menos repetitivo o de hábito" (Jaeggi 2015, 17), que pueden entenderse sobre el trasfondo de una esfera de significados socialmente constituidos. Asimismo, las prácticas no serían solo actos intencionales, sino patrones constituidos por nuestras acciones y, además, su condición de posibilidad. Como tienen un carácter habitual, estas tienden a funcionar bajo un conocimiento implícito, es decir, en la medida en la que no son problematizadas y, por lo tanto, explicitadas, funcionan muchas veces a modo de un trasfondo tácito que justifica lo que hacemos. Son también constituidas como tales solo a través de interpretaciones: un acto será comprendido en relación con otras prácticas e interpretaciones (como la comprensión de determinados conceptos); se encuentran reguladas por normas (organizadas alrededor de expectativas normativas, de la idea sobre qué significa "cumplir" con determinada práctica y hasta incluso podríamos cuestionar si es que una práctica social se encuentra inevitablemente implicada en una forma de vida); $y$, finalmente, se encuentran

I Como se diferencia, por ejemplo, la elección de pegarle a un hijo frente a la decisión que podría tomar uno de comer plátanos (Jaeggi 20I5, I5). 
dirigidas a un fin u objetivo (Jaeggi 2015 17-18). Jaeggi se referirá al "conjunto" de prácticas sociales como aquellas que “(...) abarcan una diversidad de prácticas que se encuentran relacionadas entre sí sin construir una totalidad cerrada e impenetrable" (2015, 18); de ahí que puedan ser criticadas, ya que comparten un horizonte común de interpretación, es decir, funcionan sobre un trasfondo de constitución social de significados.

Podemos determinar de forma más precisa la noción de "prácticas sociales" y el criterio normativo comunitario propiamente lingüístico que recae sobre ellas a partir de las tesis sostenidas por Robert Brandom en su artículo "Freedom and Constraint by Norms" (1979). Lo que hace que una performance lingüística sea correcta o incorrecta o que una declaración sea apropiada o inapropiada es una práctica social que determina las normas, que constituye el lenguaje. Al igual que Jaeggi, se refiere a las prácticas sociales como resultados de la acción humana que son inherentemente normativos: "Especificar una práctica social es solamente especificar qué se toma en cuenta cuando la comunidad responde a algún acto o enunciado como a un correcto funcionamiento de dicha práctica. Los criterios de identidad para las prácticas sociales apelan al juicio de la comunidad (donde "juicio" aquí no debe tomarse como que implica que la respuesta sea una evaluación verbal explícita). Lo que la comunidad dice o hace tiene que ver con la corrección que refiere a sus propias actuaciones y prácticas. Clasificar el comportamiento de una comunidad de esta manera en prácticas sociales de acuerdo a las respuestas con criterios complejos es algo que hacemos desde el exterior, como parte de un intento de comprenderlos" (Brandom 1979, 188).

De esta forma, se desarrolla una noción de libertad al establecer una distinción social y no ontológica entre hechos y normas. La diferencia entre ambas no sería una diferencia objetiva; antes bien, se basaría en el modo en que una comunidad trata o reacciona ante el comportamiento de una persona. La normatividad será una condición necesaria para la libertad; pero esto no quiere decir que uno se someta a una mera repetición de normas, sino que sea capaz incluso de crearlas. De esta forma, Brandom reinterpretará la crítica de Hegel a Kant, complementando la concepción kantiana de libertad con la hegeliana ${ }^{2}$. Las (normas y hechos) y la interpreta como una diferencia social y no objetiva. En ese sentido, la 
similitudes que esto guarda con la tesis de Jaeggi son bastante evidentes. En principio, participamos de prácticas sociales - muchas veces sin saberlo- que se materializan en instituciones $y$, en general, en estructuras materiales que nos posibilitan actuar o hacer las cosas de determinada manera (Jaeggi 20I5, 16)3. Así también, existe un criterio de corrección basado en desenvolvimientos pasados que ya han sido aceptados por la comunidad (Brandom 1979, 189) ${ }^{4}$ y, por lo tanto, existe también la posibilidad de someter dichas prácticas a dinámicas de cambio cuando vemos que lo que se ha tomado por seguro ha sido desestabilizado. Si bien Brandom se refiere a expresiones lingüísticas y conceptos, hay que tener en cuenta que estos tienen repercusiones materiales, como se verá más adelante en conexión con los problemas de segundo orden que presentará Jaeggi.

No obstante, la tesis central del artículo de Jaeggi refiere a un sentido pragmatista de las formas de vida. De esta manera, las formas de vida serían instancias de solución de problemas, en donde las soluciones serían las mejores posibles a los problemas específicos que se presentan. En este punto es preciso explicar a qué se refiere Jaeggi con "problemas". En primer lugar, son propiamente problemas aquellos conflictos complejos o dialécticos que ocurren al interior de las formas de vida; a estos se les llama "problemas inmanentes de segundo orden", y son los que dan pie a enfatizar la dinámica de cambio y conflicto contenida en las dificultades y posibilidades de crisis que suscitan las tareas $y$ estrategias específicas diseñadas para resolver ciertas dificultades inmediatas (Jaeggi 2015, 22-23). Más aún: “Los problemas son desarrollados históricamente, formados culturalmente y "predefinidos normativamente". Aparecen en el contexto de una forma de vida que ya se encuentra históricamente situada y socialmente institucionalizada. Se determinan y forman en un contexto sociocultural particular; solo aparecen en el contexto de una situación que ya ha sido interpretada" (Jaeggi 2015, 22).

Es así que los problemas concernientes a las formas de vida son problemas de segundo orden, que tienen que ver con recursos culturales-conceptuales que

libertad de un individuo se encontrará supeditada a las normas inherentes de una práctica social, que es precisamente lo que Hegel plantea con respecto a la libertad (Brandom 1979, 187).

3 La normatividad de las prácticas posibilitan la acción.

4 A esta idea se refiere Jaeggi con el "carácter de hábito" de las prácticas sociales, las cuales son patrones que se han ido constituyendo por nuestras acciones $(2015,17)$. 
una forma de vida tiene a su disposición para resolver otro tipo de problemas, que Jaeggi llamará "problemas de primer orden”. Estos últimos se dirigen a meros hechos o a la resolución de problemas materiales, mientras que aquellos se dirigen a las prácticas e interpretaciones, al ámbito de la consistencia de las razones. Para Jaeggi, estos problemas complejos, además de distinguirse de las necesidades ahistóricas ${ }^{5}$ y de los problemas de primer orden (aunque dependen de ellos), tienen dos características particulares. Por un lado, se originan a partir de las soluciones anteriores (a otros problemas de segundo orden); por otro lado, el orden normativo desde donde se sostiene la potencial crítica y superación de un problema de segundo orden trasciende el contexto cultural. De esta forma, las instituciones que surgen en un contexto "ético"6 histórico específico son soluciones a un conjunto de problemas propiciado por las antiguas formas de organización de la sociedad. Cuando estas se vuelven insuficientes para lidiar con los problemas que ellas mismas producen, se manifiestan en crisis ${ }^{7}$ funcionales y normativas. En ese sentido, aunque las crisis pueden implicar obstáculos a la satisfacción de necesidades y se evidencian en la desestabilización de la sociedad, estas requieren la transformación de la comprensión de dichas instituciones para ser resueltas ${ }^{8}$.

Al respecto, Brandom esbozará una noción de "libertad expresiva" que permitiría ampliar este enfoque. El aprendizaje de una lengua no se limita, según Brandom, al uso de una serie de oraciones, sino que implica también adquirir la capacidad de producir nuevas oraciones que una comunidad pueda considerar como apropiadas $y$, con ello, hacer posible la articulación de nuevos estados mentales. Sin embargo, el ejercicio de esta libertad expresiva necesitaría de un contexto sustantivo: "La libertad expresiva consiste en la generación de nuevas posibilidades de actuación que no existían y no podían existir fuera del marco de las normas inherentes en las prácticas sociales que constituyen el lenguaje. Uno adquiere la libertad de creer, desear, e intención de que existan

5 Según Jaeggi, los problemas parten de las formaciones culturales mismas y no habría que confundirlos, por ejemplo, con la noción de "capacidades" propuesta por Martha Nussbaum $(2015,22)$.

6 Es decir, un trasfondo valorativo en común.

7 Jaeggi utiliza el concepto de "crisis" para referirse a los problemas suscitados por las propias estructuras de las formas de vida $(2015,14)$.

8 Para esclarecer esto, Jaeggi desarrolla el ejemplo de la teoría de la familia de Hegel en su artículo "Towards an Immanent Critique of Forms of Life" (2015, 23-24). 
nuevos estados de cosas solo en la medida en que alguien habla un idioma u otro, en que se encuentra constreñido por algún complejo de normas sociales” (Brandom 1979, 194).

La fuerza para resolver problemas de segundo orden de las formas de vida estaría ligada tanto al nivel de comprensión de ciertos conceptos como a la capacidad para articular otros nuevos; es decir, al nivel de destreza para generar nuevas interpretaciones. Por ello, la crítica, si ha de ser inmanente, debe estar enfocada en la identificación de este tipo de problemáticas. Esto será posible a través de una explicitación de los recursos conceptuales de una sociedad en conexión con otros conceptos, cuyas repercusiones son materiales. El enfoque inferencialista de Brandom aporta los recursos necesarios para entender con mayor precisión este último punto?.

\section{§ 3. Los problemas de segundo orden a la luz del enfoque inferencialista de Brandom}

En el primer capítulo de su libro Articulating Reasons (2000), Robert Brandom retoma el debate entre dos concepciones de lo conceptual: inferencialista y representacionalista. Dicho debate gira en torno al origen y la justificación de nuestras ideas, en otras palabras, a la explicación sobre qué significa que algo sea entendido como la representación de un estado de cosas. Es así que la teoría epistemológica moderna, que planteaba una noción meramente ostensiva del lenguaje (podemos hablar de Descartes y de los empiristas británicos), proponía que existía una verdad aislada en la representación: una proposición era verdadera cuando correspondía a una realidad material, a un estado de cosas sin la necesidad de la asociación de aquella proposición con otras.

Por otro lado, los inferencialistas buscaron definir las propiedades representacionales en términos de inferencias, las que debían ser entendidas con anticipación o como un nivel previo: la verdad y la representación es entendida solo

9 Gianfranco Casuso también desarrolla el aporte de Brandom a Jaeggi en relación con la posibilidad de una crítica inmanente a partir de la experiencia de la disonancia cognitiva generada por la exclusión en su artículo "Power and dissonance: Exclusion as a key category for a critical social analysis" (2017, 608-622). 
en conexión con otras ideas que no son evidentes o manifiestas, y que tienen un papel importante en el razonamiento. Brandom reconstruye las posturas de Sellars y de Hegel, que establecen que incluso en el caso de relaciones no-inferenciales es preciso el establecimiento de inferencias y proponen así un holismo conceptual. En su artículo "Knowledge and the Social Articulation of the Space of Reasons" (1995), aparece un ejemplo sumamente ilustrativo sobre lo que significaría entender un concepto bajo esta última perspectiva. Tomemos el caso de un loro que puede decir "esto es rojo" en respuesta a un estímulo suscitado por algo que, en efecto, es rojo. La interrogante gira en torno a si se puede poner en el mismo nivel del loro a un hombre que responde de la misma manera al mismo estímulo. ¿Es el loro igual que el hombre en este sentido? ¿Qué significa, en todo caso, que algo sea rojo? (Brandom 1995, 896-897).

Este ejemplo dará pie a la explicación de lo que es el espacio de razones y, de esta forma, a la diferencia entre sentience ${ }^{10}$ y sapiencia. La capacidad que hace posible que el loro pueda decir "esto es rojo" se debe únicamente a la sentience, esto es, a la capacidad de responder diferencialmente ante estímulos sensibles de manera fiable (algo que el hombre también está en capacidad de hacer). No obstante, en el hombre hay un proceso adicional que lo distingue del loro, que consiste en poder justificar inferencialmente por qué "esto es rojo": "porque tiene un color", "porque no es azul”, etc. La noción de razonamiento que propone Brandom refiere, pues, a la capacidad de establecer relaciones de inferencia entre un concepto y otros. Entender un concepto significa saber establecer sus relaciones con otros conceptos. Esto hace que la diferencia entre la sentience y la sapiencia sea normativa y no natural, de modo que se aleje del error del representacionalismo de hacer del significado un componente no-inferencial, supuestamente aislado de la red de conexiones que hacen una proposición posible: "Es en este punto en el que Sellars introduce su pensamiento central: para que una respuesta tenga contenido conceptual, debe jugar un rol en el juego inferencial de realizar afirmaciones y dar y pedir razones. Comprender o entender un concepto es tener dominio práctico sobre las inferencias que se encuentran implicadas -saber, en el sentido práctico de 
ser capaz de distinguir (un tipo de know-how), qué se sigue de la aplicación de un concepto y de dónde se sigue ese concepto" (Brandom 2000, 48).

Brandom establece, además, una defensa de lo que Sellars llamó "inferencias materiales", en contra de la identificación de la articulación inferencial con la articulación lógica. Restringir la noción de "lógico" en el sentido de identificar la fuerza lógica de las razones con inferencias formalmente válidas es un error que omite un compromiso sustancial o fundamental (Brandom 2000, 53). El problema con esta concepción es que reconoce las buenas o malas inferencias (pretensiones de validez) en virtud de la forma, cambia la bondad primitiva de la inferencia por la verdad de los condicionales. Como ejemplo podemos considerar la inferencia de la proposición "Pittsburgh está al este de Princeton" derivada de la proposición "Princeton está al oeste de Pittsburgh" (Brandom 2000, 52). Son, pues, los contenidos de los conceptos "oeste" y "este" los que hacen que la anterior sea una buena inferencia.

La posterior apertura de un espacio de razones permite observar que las aplicaciones de los conceptos son tomadas o tratadas como correctas de acuerdo con las razones que prevalecen en el contexto, lo cual permite, a su vez, una crítica historizada. Volver a la noción de "problemas" de Jaeggi nos ayudaría a entender este punto. Según esta, los problemas son dados y producidos. Son dados en el sentido en que una situación indica que se está produciendo una crisis que no puede ser ignorada (se propicia un nuevo problema); y son producidos en la medida en que existe una identificación de un problema como una interpretación no pertinente para el contexto problematizado (Jaeggi 2015, 25).

Complementar la concepción de problema de Jaeggi con la perspectiva inferencialista de Brandom, nos permite referirnos a una superación (en el sentido hegeliano de la palabra) de contenidos conceptuales, es decir, darle soluciones a problemas de segundo orden (dentro del orden de lo cultural-conceptual). Solo a partir de la constante apertura a la revisión de contenidos conceptuales se evitará caer en lo que Brandom llamará semantic naiveté. De acuerdo con el autor, esta expresión se refiere a tomar por sentado los contenidos 
conceptuales" de las actitudes cuyas relaciones racionales la genealogía ha tratado de disolver $(2012,6)$. Esto es lo que Jaeggi llama "proceso dinámico".

Tanto la condición de posibilidad de los problemas de segundo orden como su resolución están basadas en la idea de que, para apreciar la interdependencia entre compromisos semánticos (escoger un lenguaje, conceptos) y epistémicos (tomar ciertos enunciados por verdaderos) ${ }^{12}$, los conceptos deben ser categorías dinámicas y no categorías estáticas: "Nuestros juicios moldean nuestros conceptos no menos de lo que nuestros conceptos moldean nuestros juicios" (Brandom 2012, 12). Así, pues, el inferencialismo de Brandom nos brinda una imagen más precisa de la resignificación de valores y fines (conceptos morales) que supone la crítica inmanente de Jaeggi y su relación con la praxis.

\section{§ 4. La apertura del espacio de razones y la actualización semántica de una crítica inmanente}

Los compromisos con el lenguaje de los que habla Brandom tienden a funcionar de manera implícita; se trata de aquello que subyace y que solo se hace evidente cuando alguien tiene que dar cuenta del uso de un concepto aislado refiriéndose a otro concepto. Brandom llama al esclarecimiento de dichos compromisos "método socrático", en alusión a un proceso de racionalización de algo que era tácito; esto posibilita no solo la explicitación de lo inadvertido, sino además el reconocimiento de los errores inferenciales del interlocutor. Se apela así una forma de racionalidad centrada en la noción de "expresión”, en contraste con la noción instrumental de racionalidad: antes que la adecuación o falta de adecuación entre los medios que la razón traza para sus fines, el criterio de racionalidad aquí en juego se basa en la capacidad para explicitar en enunciados aquello que funciona como el trasfondo semático-conceptual implícito de nuestras acciones. Esto posibilitará una crítica semántica que puede tener injerencias relevantes en las formas de vida y respectivas prácticas sociales características de nuestra relación con otros: "El método socrático es una forma de llevar nuestras prácticas bajo control racional expresándolas de

II Considerar que lo epistémico puede ir separado de lo semántico y de lo pragmático.

12 Podríamos llamar al cambio de interpretación de un concepto por una interpretación mejor como una "acumulación de experiencias", en términos de Jaeggi. 
forma explícita en una forma en la que puedan ser confrontadas con objeciones y alternativas, una forma en que puedan ser exhibidas como las conclusiones de inferencias buscando ser justificadas sobre la base de premisas desarrolladas en razones, y como premisas en posteriores inferencias explorando las consecuencias de aceptarlas" (Brandom 2000, 56).

$\mathrm{Si}$, entonces, la corrección de una inferencia no es puramente formal, pues son los contenidos de estas mismas inferencias los que determinan su validez (ya no en términos formales), ¿cuál es el estándar de evaluación de una inferencia material? ¿Existe algún criterio objetivo para evaluar la validez de una inferencia material? Brandom responde a esta pregunta al sostener que la conexión entre la significación pragmática y el contenido inferencial es proporcionada por el hecho de que afirmar un enunciado implica asumir un compromiso con la corrección de la inferencia material en sus circunstancias y consecuencias de aplicación, es decir, responder diferencialmente de acuerdo a las circunstancias de la aplicación apropiada de un concepto y distinguir las consecuencias inferenciales propias de aquella aplicación $(1994,120)$ o entender el concepto.

El método socrático desarrollado por Brandom nos lleva a reconocer dos aspectos importantes de su propuesta: por un lado, existe una articulación entre verdad, fiabilidad y justificación; por otro lado, se hace patente el rol intersubjetivo del hacer explícito, la necesidad de una figura que interpela. Ahora bien, hasta este momento se ha hablado de inferencialismo en conceptos y en juicios o enunciados de forma indistinta. Pero para tener una mejor comprensión acerca de este tema, es necesario advertir la recuperación de una idea kantiana por parte de Brandom: la unidad de sentido más pequeña por la cual podemos hacernos responsables es, precisamente, el juicio. Es así que podemos entender el juicio, y posteriormente la acción, como aplicaciones de conceptos (Brandom 2000, 80). Las distinciones entre juicios (entendidos como razones para la acción) expresan compromisos con el lenguaje: tenemos que responder por el uso de una determinada articulación de conceptos debido a que nuestro derecho al establecimiento de dichos compromisos siempre se encuentra en discusión, y estos solo serán considerados racionales cuando brindemos razones o justificaciones por medio de inferencias.

Brandom retomaría la relación entre verdad, justificación (o el sostener un derecho a un compromiso) y creencia (entendida como el sostenimiento de 
un compromiso en un espacio lógico de razones, es decir, en la dinámica social de dar y recibir razones). Más aún, las condiciones de verdad se tomarán en cuenta a través de la diferencia entre atribuir un compromiso a otro y asumir ese mismo compromiso uno mismo. Es así que el conocimiento requiere tres diferentes actitudes: atribuir compromisos, atribuir derechos y asumir compromisos. El elemento esencialmente social de este espacio de razones puede ser explicado de una forma más pertinente con la interpretación que hace Brandom de la concepción hegeliana de la autoconciencia como un logro de naturaleza social: "La autoridad para hacerse a uno responsable (de lo que uno piensa o lo que uno hace) tiene sentido solo en un contexto en el que uno puede ser considerado responsable (...). Los estatus normativos, como autoridad y responsabilidad, y los mismos que son sujetos de aquellos estatus, Hegel enseña, se encuentran instituidos por reconocimiento recíproco: esto es, por individuos tomándose o tratándose prácticamente como autoritativos y, por lo tanto, responsables" (Brandom 2012, 13).

Al articular, pues, esta idea del conocimiento con el inferencialismo podemos decir que los reportes no-inferenciales (como la percepción) son productos de dos capacidades diferenciables: una disposición fiable a dar respuestas diferenciales, así como la capacidad de tomar una posición en el juego de dar y recibir razones. Es la segunda la que permite colocar alguna aseveración en el espacio de razones y evaluar su justificación, y así dar pie a una posible crítica' ${ }^{13}$.

Rainer Forst, en su artículo "Noumenal Power" (2015), transforma el espacio de las razones (epistémico) a uno práctico-político. Para él, el poder no se encuentra en la esfera fenoménica (no se mide por sus efectos), sino en la esfera noumenal, es decir, de la posibilidad ${ }^{14}$. Es allí donde introduce el espacio lógico de las razones, en donde se es capaz de justificar lo que uno dice: “El espacio noumenal que creo es relevante aquí es un espacio 'impuro' que incluye lo que las personas ven como justificado por buenas o malas razones. Necesitamos criterios para distinguir las dos, pero el concepto general de poder

I3 El uso de la teoría de Robert Brandom para la crítica social es también desarrollado por Gianfranco Casuso (2017). La fuente que utiliza para tal desarrollo es, hasta cierto punto, la lectura de Rainer Forst (Casuso 2017, 608-622).

14 "El ejercicio y los efectos del poder están basados en el reconocimiento de una razón -o mejor y con más frecuencia, varias razones- para actuar de manera distinta a la forma en que uno hubiera actuado sin esa razón" (Forst 2015, I16). 
no contiene esos criterios" (Forst 20I5, II2-II3). De esta forma, el análisis del poder consistiría en gran parte en el “(...) análisis discursivo de justificaciones hegemónicas o dominantes que se materializan en un orden social como un orden de justificación" (2015, 124). En ese sentido, todo orden social es un orden normativo y cada una de estas entidades puede definirse. La forma en que opera dicho contenido es precisamente en el orden de la justificación, es decir, cada orden social ofrece razones para actuar de determinada manera. Esto también se encuentra en Jaeggi cuando define las formas de vida.

Al respecto, es preciso mencionar que no toda explicitación tiene una función crítica ni de transformación. De acuerdo con Brandom y Forst, esta solo abre el espacio de razones y las posibilidades son dos: puede haber una transformación social o puede haber una perpetuación del estado actual de las cosas con mejores razones. Tampoco para Jaeggi podemos hablar de una "ineludibilidad del progreso" 15 , pues el progreso moral relacionado con una transformación social está supeditado a las circunstancias de crisis y resolución de las mismas: "Las formas de vida son exitosas cuando pueden ser entendidas como el resultado de procesos exitosos de acumulación de experiencias (o un proceso de aprendizaje) y cuando permiten mayor aprendizaje" (Forst 20I5, 26). No obstante, la apertura del espacio de razones, puesto en vinculación con las problematizaciones de las formas de vida, permitiría una actualización semántica de la crítica inmanente; de este modo, sería posible reconocer los procesos de racionalización (involucrados en la resignificación conceptual de la crítica inmanente) como procesos de determinación de contenidos conceptuales en dos registros lingüísticos. En el primero se descubre qué son los conceptos por medio de las articulaciones de razones esencialmente retrospectivas (atender a la interconexión semántica). En el segundo se determina el campo semántico de los conceptos, lo cual se manifiesta en las nuevas interpretaciones de los mismos (configuración provisional del significado).

En suma, podría decirse que una crítica inmanente es una crítica intrahistórica ${ }^{16}$, que toma los ideales vigentes de una sociedad dada para confrontarlos con la

I5 Título del artículo de Axel Honneth en referencia a Kant (2009, 9-25). Sobre el progreso existe un debate actual en la teoría crítica (Allen 2016; Allen y Mendieta 2018).

16 “Deja el terreno de lo propiamente 'ético' y entra en un análisis de los entramados inertes de la práctica de acuerdo a la normatividad de su posición dentro de un proceso histórico de resolución de problemas" (Jaeggi 20I5, 26). 
realidad existente (es decir, el entramado semántico que soporta el uso de los conceptos) en la que supuestamente se habrían realizado. La crítica inmanente obtiene una determinación más precisa si tomamos los problemas de segundo orden ${ }^{17}$ como la ocasión para revelar y evaluar las inferencias conceptuales (o el entramado semántico) allí en juego, pero en última instancia sometidos a una indeterminación interpretativa ${ }^{18}$. Según Jaeggi, este proceso implica también abrirnos a un "pluralismo experimental", en el que tengamos en cuenta que no existe una forma de vida que no resuelva problemas sin crear nuevos $y$ en donde la comparación entre unas y otras permita una experimentación para encontrar soluciones aceptables a nuestros problemas $(2015,27-28)^{19}$. La libertad, pues, sería la apertura permanente al espacio de las razones, es decir, la capacidad de revelar y cuestionar los vínculos inferenciales entre nuestras creencias y razones (Forst 2015, I19-120) ${ }^{20}$, así como de ser capaces de representarnos un orden de cosas alternativo que evite la reproducción de lo ya existente ${ }^{21}$.

\section{Bibliografía}

Allen, Amy, 2016. The End of Progress: Decolonizing the Normative Foundations of Critical Theory. New York: Columbia University Press. https://doi.org/I0.73/2/allel7324

Allen, Amy y Eduardo Mendieta, 2018. From Alienation to Forms of Life: The Critical Theory of Rahel Jaeggi. Pennsylvania: Penn State University Press.

Brandom, Robert, 1979. Freedom and Constraint by Norms. American Philosophical Quarterly 16 (3), 187-196.

17 La detección de contradicciones entre realidad efectiva de una institución y las metas y fines que debería cumplir, que es el trasfondo normativo que Jaeggi busca explicitar.

I8 "Es inmanente porque toma su punto de partida refiriéndose a las crisis inmanentes y a la erosión de las prácticas sociales e instituciones. Es transformativa porque la evaluación de los procesos de resolución de problemas permite trascender el contexto -e inicia el cambio" (Jaeggi 20I5, 27).

19 Esto distingue a Jaeggi del pluralismo de la "abstinencia ética". Las formas de vida no son mónadas aisladas las unas de las otras, sino más bien una pluralidad de experimentos para la resolución de problemas (Jaeggi 2015, 28).

20 Brandom también habla de un tipo de libertad similar que requiere la capacidad de regirse por normas conceptuales $(2012,13)$.

21 La imposibilidad del acceso a la explicitación de las razones y de sus vínculos inferenciales es lo que constituye en gran parte la exclusión (Casuso 2017, 615). 
- 1994. Making It Explicit. Reasoning, Representing, and Discursive Commitment. Cambridge: Harvard University Press.

- 1995. Knowledge and the Social Articulation of the Space of Reasons. Philosophy and Phenomenological Research 55 (4), 895-908.

- 2000. Articulating Reasons. An Introduction to Inferentialism. Cambridge: Harvard University Press.

- 2012. Reason, Genealogy, and Hermeneutics of Magnanimity. En: http://www.pitt. edu/ brandom/currentwork.html. Consultado julio del 2016.

Casuso, Gianfranco, 2017. Power and dissonance: Exclusion as a key category for a critical social analysis. Constellations 24 (4), 608-622. https://doi.org/I0.IIII//467-8675.12289

Forst, Rainer, 2015. Noumenal Power. The Journal of Political Philosophy 23 (2), III-I27. https://doi.org/I0.1 III/jopp.12046

Honneth, Axel, 2009. Una patología social de la razón. Sobre el legado intelectual de la Teoría Crítica. En: Patologías de la razón. Historia y actualidad de la Teoría Crítica. Buenos Aires: Katz, 27-52.

Jaeggi, Rahel, 2015. Towards an Immanent Critique of Forms of Life. Raisons politiques I (57), 13-29. https//doi.org/10.3917/rai.057.0013 